\title{
Partnering Care Delivery and Research to Optimize Health
}

\author{
Laura A. Coleman, PhD; Thomas E. Kottke, MD, MSPH; Brian Rank, MD; Douglas J. Reding, MD, MPH;
} Mark Selna, MD; George J. Isham, MD, MS; Andrew F. Nelson, MPH; and Robert T. Greenlee, PhD, MPH

\begin{abstract}
A close partnership between care delivery and research organizations has the potential to provide essential elements needed to optimize health and health care. This clinical leadership panel, held during the 14th Annual Health Maintenance Organization Research Network (HMORN) Conference, identifies the value, opportunities and challenges of those close partnerships between three HMORN care delivery and research organizations. The objectives of this plenary session were: $(\mathrm{I})$ identify the important facets of partnership that bring value to care delivery and research, (2) pinpoint the critical alignments of care delivery and research that are needed to fulfill the promised value between clinical and research organizations, and (3) recognize the challenges that clinical and research organizations need to address.
\end{abstract}

Keywords: Clinical; Health care; Research

his paper explores the junction of research and clinical Increasingly, bi-directional linkage of practice and research is viewed as an advantage for health care organizations; the ability to create a successful relationship is critical to their positioning for competition in the modern world.

The challenge that health care organizations face going forward is leveraging the full capacities and data assets of medical practices, health plans, research units, hospitals, and of course, consumers, to create new strengths and capabilities. Critical to this challenge is the ability of research to help identify the optimal ways for health care organizations and medical groups to position themselves in order to achieve their mission. Today, this mission can be referred to as the "triple aim:" maximizing patient experience, making sure we have outstanding clinical outcomes, and explicitly addressing the affordability of health care. The only way to achieve all three parts of the mission is collaboration. practice, and its important role in optimizing health care.

With these thoughts in mind, a panel of clinical leaders from three integrated, large, health care financing and delivery organizations were asked to share and discuss their comments in view of a recent article offering a new perspective for optimizing health care through research. ${ }^{1}$ The author of that paper was the introductory speaker and he presented five broad principles that could guide future work on this challenging problem. Underlying Dr. Kottke's main points is a call to move the goal of health sciences research away from merely the pursuit of knowledge and its 'translation' into clinical practice, and instead toward a partnership that optimizes clinical practice through research in order to improve the health of the population. ${ }^{1}$

\section{Tom Kottke}

The five principles for optimizing care through research are summarized in table 1 . The first key principle is that the needs of patients and populations should determine the research agenda. Heart disease serves as an example of the importance of asking whether a particular line of research is

Received: November 18, 2008

Accepted: November 18, 2008

Reprint Requests: Laura Coleman, PhD, Epidemiology Research Center, Marshfield Clinic Research Foundation, 1000 North Oak Avenue, ML2, Marshfield, WI 54449, Tel: 7I5-389-3350, Fax: 7I5-389-3880, coleman.laura@mcrf.mfldclin.edu 
Table 1. Key principles for optimizing health care through research 1

Principle 1 The needs of patients and populations should determine the research agenda

Principle 2 The research agenda must address contextual and implementation issues

Principle 3 The research agenda must determine the methods, rather than the methods determining the agenda

Principle 4 Researchers and clinicians must collaborate to define the research agenda and resources, and implement the findings

Principle 5 The level of funding for implementation research needs to be commensurate and proportional to the magnitude of the task

likely to benefit our patients. Research has shown that for heart disease, the way in which health plans can have the greatest impact on preventing or postponing deaths is by reducing the prevalence of disease via nutrition, physical activity, and adherence to medications. ${ }^{2}$ The evidence is clear, for example, that if we maintain patients on aspirin, beta blockers and statins, we can reduce mortality by $85 \%$ to $90 \% .^{3}$ The majority of deaths are caused by a few common behaviors (poor diet, inadequate physical activity, tobacco use and exposure and hazardous drinking); even the most comprehensive research programs into rare causes of disease or rare diseases will have only limited impact. If researchers are going to have an impact, we have to conduct research in nutrition, physical activity, and smoking cessation. Going forward, descriptive epidemiology should set the agenda for basic biomedical research.

The second principle for optimizing practice through research is that the research agenda must address contextual and implementation issues, including the development of delivery and accountability systems. As an example, many people think that the rise of surgery was due to anesthesia, but it was not. Rather, the rise in surgical success was the result of implementing delivery and accountability systems for maintaining good hygiene. Achieving quality improvement involves setting it as a priority, followed by changing systems to make it happen.

A third key principle is that the research agenda must determine the research methods, rather than the methods determining the agenda. We have to decide, as a country, whether a particular research area is a priority. As an example, work by Ballard et $\mathrm{al}^{4}$ on improving the delivery of clinical preventative services led to the implementation of multiple initiatives in Texas to increase clinical preventative service delivery, and as a result, they were able to increase "perfect" care from $20 \%$ to $50 \%$ over a period of 5 years.

A fourth important principle is that researchers and clinicians must collaborate to define the research agenda and resources, and implement the findings. A strategy that works is for researchers and clinicians to sit down beforehand and discuss what evidence is needed in order to implement an intervention. Moreover, a level of funding is needed for implementation research that is commensurate and proportional to the task. The task needs to be reframed to keep the research agenda focused on improving health and on acknowledging that the effort needs to be more comprehensive than translating research into practice. We suggest that the task be reframed as "optimizing practice through research" so that clinical practice is viewed as the client and research is viewed as the tool.

And finally, the last principle is that the level of funding for implementation research needs to be commensurate and proportional to the magnitude of the task. In order to get full value from the research that has already been completed, we need the resources to take that work one step further and incorporate innovation in clinical practice to improve patient care.

\section{Mark Selna}

I agree whole-heartedly with principle one, that the research agenda needs to reflect the needs of patients and populations. The first of three challenges that I see with this principle is that a patient's true needs are generally not known - whether it be the usual paternalistic symptom-focused interaction between patients with their physicians or the even further academic discussions among health services researchers. A second challenge is refining those needs in a way that they actually reflect the broader needs that the payers have as well. Patient needs have to be met in a way that is economically sustainable. The third challenge is delivering against the disparate needs of individual patients. We want to avoid the experience of optimizing for populations, while inadvertently sub-optimizing for every patient in the population.

Principle two, the notion that we should address both the context and implementation issues of research, should perhaps be our first objective. The challenge lies in determining how to accomplish this. Perhaps we should focus more on operations than on biology. The notion that care management is not standardized, and as a result, is not amenable to research, I believe is a fallacy. Care management, while often very dynamic and requiring frequent and intense monitoring, can be standardized and, as such, can be studied. A primary operational issue and therefore research design consideration, is viewing the clinical care process as one of perpetual care as opposed to unrelated episodes of care; unfortunately, the level and scope of care plan development execution and oversight in our industry is woefully inadequate. 
Principle three, that research agendas need to dictate the methodologies and not vice versa, is absolutely true in order to conduct true effectiveness research (as opposed to efficacy research). Important challenges in this regard are to avoid relying too narrowly on literature-based findings, and on not doing enough pre-implementation operational due-diligence. As such, it is practical, pragmatic, and ultimately less expensive to take the extra time to conduct some rapid-cycle prototyping, in the operational environment, in order to refine the operational/study design and to co-opt the necessary operational and clinical stakeholders. Ultimately, we get better research as a result.

Principle four relates to the importance of collaboration between researchers and clinicians to develop a research agenda. There is a lot of merit to moving away from a purely government-defined research agenda to one that involves clinicians, but I would submit that we as clinicians are not terribly motivated to innovate, nor are we motivated to rationalize the operational infrastructure enabling what we do. The "end user" from a translational research standpoint is not the clinician, but rather, is the patient, employer and payer. Consequently, the collaboration should be broader than simply being between researchers and clinicians.

Lastly, principle five - that funding for implementation research be commensurate and proportional with the magnitude of the task - I agree whole-heartedly. There are, perhaps, a few caveats, one being that we need to challenge people to examine the financial rationale, or economic return that justifies making an investment. This determination, for a provider organization, is not as simple as it is for health plans, where it is a dollar for dollar return on investment. Instead, we need to consider the measurable returns on the dollar investment, which can include health benefits, dollar benefits, or some other type of benefits. It is a challenge for people to think about benefits quantitatively.

Another caveat is the need to build the data capture process and the data analytic process into the operational redesign effort. Without fully knowing what research agenda might result from the redesign work, we know that better data sets, both in scope and integrity, can help drive research.

The last caveat is the importance of conducting research at a lower per-event acquisition cost by taking advantage of natural platforms, natural stakeholder groups, funding opportunities, and pay-for-performance opportunities that are inherent to the health care delivery system. Decreasing reliance on governmental sources of funding and diversifying funding will be an advantage in the future.

In closing, ideally, research should be focused on eliciting, understanding, and prioritizing a person's needs, risks and preferences, as well as their capacity to engage over the long term. We need to understand how to obtain the data, how to capture and interrogate it in real-time, how to do so at minimal cost, how to programmatically generate novel associations in the data, and then how to deploy decision support. Lastly, while controversial within our industry but intuitively obvious to outsiders, is the need to re-design highcost components out of our core processes.

\section{Doug Reding}

While the five principles are very thought provoking, there are some inconsistencies with how clinical practice actually functions. This difference presents some challenges when trying to optimize clinical care through research.

For example, early on in our training, scientists are taught about the scientific method, with a focus on conducting hypothesis-driven research. In practice, however, the scientific world is extremely competitive with $<15 \%$ of all grant applications funded, and with the types of research that are funded largely dictated by the funding agency. For this reason, researchers should ideally pursue resources that will allow us to set our own agenda. Basic science research may be the link to true discovery going forward; this can be thought of as 'incremental research discovery.' Rarely does a single scientific experiment lead to significant cures in the health care industry. In clinical practice, in order for a new process to be implemented, findings from well-conducted scientific studies are needed.

Regarding the notion that the agenda dictate the analysis, this is also an interesting point. Consider the fact that $80 \%$ of health care expenditures are spent on $10 \%$ of people in the last 6 months of their lives. The scientific research agenda in the U.S. does not allocate a proportionate amount of money, time or effort on those last 6 months of life. We rarely ask clinical questions that are important to manage that $80 \%$ of health care at the end of lives. Providers have an obligation to address some of the chronic health problems, particularly if $25 \%$ of excessive care being delivered is futile.

In a patient-centric model, chronic disease management is the priority, with a particular focus on delivery systems and methodologies in order to improve quality and process for health care. If this focus is going to be our priority going forward, there are some important questions that need to be addressed: what partnerships are important? How should the system be aligned, both at an institutional as well as a national level? To answer these questions, the health care delivery system needs to be viewed as a science experiment, emphasizing principles of standardization, quality control, cost containment, and improved value to patients and clients.

At the present time, we do not have a good system in place for translating scientific discoveries and results to patients. Information systems provide the opportunity for seamless integration of information into practice. The notion that electronic medical records will increase efficient delivery of care, standardize the content of care, and improve the messages to the patients is vital and crucial. Information 
systems can also aid in decision support. For these reasons, we need to align the interests of information systems, medical genetics, and personalized health care in order to create a medical system that can meet the needs of patients.

\section{Brian Rank}

As care systems, our goal is to deliver 'perfect care' to each of our patients and hence 'perfect care' to the populations we serve. 'Perfect care' refers to delivering all the care that can help - and only the care that can help - customized to the needs, preferences, and values of each patient.

The Institute of Medicine (IOM) defines health as the degree to which health services for individuals and populations increase the likelihood of desired health outcomes and are consistent with current professional knowledge. The World Health Organization (WHO) defines health as a state of complete physical, mental, and social well-being and not merely the absence of disease or infirmity. In the IOM report on crossing the quality chasm, which documents many of the deficiencies of the health care of Americans today, it notes a peculiar statistic. ${ }^{5}$ There is a 17 -year gap between when new knowledge is created in randomized trials and when the average American finally receives the benefit of that knowledge. And even then, application of that knowledge is highly unreliable.

Research by McGlynn et $\mathrm{al}^{6}$ has suggested that Americans receive, on average, approximately one-half the amount of health care that can help them, including preventative services, acute care, or chronic conditions. On the other hand, our present system rewards overuse of potentially harmful and dangerous health care services. Clearly the present health care system in the U.S. is not designed to reliably bring the right care and support to the right patient at the right time. That is both an indictment and a promise for research on health care delivery. There is evidence that organized and integrated care systems such as HealthPartners, Kaiser Permanente, Geisinger, and Marshfield Clinic do a better job than most; there still exists a huge chasm between the care that we deliver today and that which we could and want to deliver to our patients.

Reasons for this chasm are well-documented. A huge number of randomized clinical trials will be published this year, and to incorporate those into the health care system requires a great deal of knowledge and an ability to understand the interfaces between systems. At present, our health care systems are poorly organized to meet the health care needs of individuals and populations. Care is overly complex, consisting of too many valueless steps and uncoordinated handovers, which slow down appropriate care and make care more unsafe.

Our care system today remains an antiquated acute care model which requires visits to the clinic or hospital in which there is little knowledge of patient needs. Ideally, our health care system would include continuous, proactive outreach, based on best science, along with a patient's biology, preferences, and values.

In the past, performance measurement in health care systems has primarily been based on retrospective measures from random chart samples from a paper medical record. This information was almost invariably untimely, inaccurate, and not granulose enough to make decisions or to influence and guide behavior. Only recently have electronic health records allowed us to report timely and accurate data for improvement purposes.

Until recently, we spent most of our research agenda on generating new knowledge, rather than on figuring out how to reliably implement new knowledge. We are at the infancy of understanding how to reliably and systematically implement care consistent with the IOM's six aims for improvement. ${ }^{5}$ In order to accomplish this, the research agenda now must include not only traditional research techniques, but also an understanding of care process variation, along with reliability, science, and human systems engineering. Unreliability means failure to meet processes over time; our ability to do that is the difference between the health care that people could receive and that which they are receiving today.

A research agenda also needs to encompass an understanding of how human biological and cultural diversity affects care. We know that measured quality of care and satisfaction with the U.S. health care service vary by race, ethnicity, language, and socioeconomic status. In our care system, we have obtained language preferences for $99 \%$ of our 400,000 patients, and $84 \%$ have trusted us with their race and ethnicity data. This database can help us to reliably understand how to customize care to the preferences and values of patients, informed by best evidence. It recognizes the deep and respectful relationship between a patient and clinician, with an appreciation of how complex care is at the point of care. Understanding why things happen the way they do at the point of care can bring researchers and clinicians together to advance a new research agenda.

The challenge then is to understand how to redesign our systems to support patients and clinicians in an effective continuous healing relationship. When patients, clinicians and researchers effectively collaborate, we better understand how to optimize practice, to create high performing teams and thus a high performing health care system. For our organization, the close integration of care and research comes from our history and is hardwired into our organizational structure. Our health plan, research foundation, and care system are cross-pollinated by researchers, clinicians, and leaders across the hospital, medical group, dental group and health plan, and our institute for medical education.

Well-respected clinicians serve in senior leadership positions within the medical group and research foundation, and senior researchers recruit and mentor young clinicians in clinical 
care and care improvement research, creating a deeper understanding of the pressures facing care teams as they seek to deliver 'perfect' care to their patients. The close relationship between clinical care and research not only accelerates improved care for our patients, it also brings in tangible benefits to the organization. It clearly helps recruit excellent clinicians and helps build more professionally rewarding careers based on outcomes for patients. The ability to improve care, outcomes, and practice efficiency is seen as an important benefit to being a physician or dentist in our care system. For us, the challenges of linking care and research more closely are probably the same as for others, and these challenges primarily involve time and money. Clinicians in efficient practices today spend most of their time and energy in clinical care, and to incorporate clinical research into an already stressed life takes both effective mentorship and financial support. To that end, in our organization we have created partnership grants to support that relationship between clinicians and researchers. To truly succeed, however, and to change the way in which health care is delivered, we need input from patients.

\section{Acknowledgments}

Funding for this conference was made possible in part from the Agency for Healthcare Research and Quality (1R13HS017518-01) and the National Cancer Institute (1R13CA128384-02), and by HealthPartners, HealthPartners Research Foundation, HealthPartners Medical Group \& Clinics, Marshfield Clinic Research Foundation, Marshfield Clinic, and Security Health Plan. The views expressed in written conference materials or publications and by speakers and moderators do not necessarily reflect the official policies of the Department of Health and Human Services, nor does mention by trade names, commercial practices, or organizations imply endorsement by the U.S. Government.

\section{References}

1. Kottke TE, Solberg LI, Nelson AF, Belcher DW, Caplan W, Green LW, Lydick E, Magid DJ, Rolnick SJ, Woolf SH. Optimizing practice through research: a new perspective to solve an old problem. Ann Fam Med 2008;6:459-462.

2. Kottke TE, Faith DA, Jordan CO, Pronk NP, Thomas RJ, Capewell S. The comparative effectiveness of heart disease prevention and treatment strategies. Am J Prev Med 2009; 36:82-88.

3. Hippisley-Cox J, Coupland C. Effect of combinations of drugs on all cause mortality in patients with ischaemic heart disease: nested case-control analysis. BMJ 2005; 330:1059-1063.

4. Ballard DJ, Nicewander DA, Qin H, Fullerton C, Winter FD Jr, Couch CE. Improving delivery of clinical preventive services: a multi-year journey. Am J Prev Med 2007; 33:492-497.

5. Committee on Quality of Healthcare in America. Crossing the quality chasm: a new health system for the $21^{\text {st }}$ century. Institute of Medicine, National Academy Press: Washington, DC; 2001.

6. McGlynn EA, Asch SM, Adams J, Keesey J, Hicks J, DeCristofaro A, Kerr EA. The quality of health care delivered to adults in the United States. N Engl J Med 2003;348:2635-2645.
Author Affiliations

Laura A. Coleman, PhD

Epidemiology Research Center

Marshfield Clinic Research Foundation

Marshfield, Wisconsin 54449

Thomas E. Kottke, MD, MSPH

HealthPartners Research Foundation

Minneapolis, Minnesota 55440

Brian Rank, MD

HealthPartners Medical Group \& Clinics

Minneapolis, Minnesota 55440

Douglas J. Reding, MD, MPH

Department of Hematology/Oncology

Marshfield Clinic

Marshfield, Wisconsin 54449

Mark Selna, MD

Geisinger Health System

Danville, Pennsylvania 17821

George J. Isham, $M D, M S$

HealthPartners

Minneapolis, Minnesota 55440

Andrew F. Nelson, MPH

HealthPartners Research Foundation

Minneapolis, Minnesota 55440

Robert T. Greenlee, PhD, MPH

Epidemiology Research Center

Marshfield Clinic Research Foundation

Marshfield, Wisconsin 54449

\section{Appendix: Question and Answer Session}

Question, George Isham, HealthPartners: In making your initial point, you talked about the incremental impact of 'perfect care' and made the point that prevention is crucial, but my question relates to the cost of pursuing perfect care in terms of waste and overuse. Are there any comments from the panel in terms of how we also answer the question of 'perfect care' at what cost?

Douglas Reding: At our clinic, the electronic medical record has a 'dashboard' for tracking the delivery of a minimum of preventative services (height, weight, serum cholesterol, etc). Information systems can help reduce redundant care, but at the same time, we have to realize what the appropriate amount of care is for a particular patient.

Brian Rank: The point of 'perfect care' is an allocation of resources issue; what is the appropriate use of resources for the right populations? We have tried to incorporate decision support at the point of care for our clinicians. One of the caveats in a discussion about crossing the quality chasm is 
transparency. Patients should be engaged in the science behind the decision making.

Tom Kottke: It may be that we will always spend $80 \%$ of our money on the last 6 months of life. But with the opportunities that are emerging with the electronic record and the Internet, and by including the employer and the employee at the work site - the major social setting for the majority of Americanswe can initiate prevention programs as the adult enters the labor force. Yearly health assessment, advice, and support for nutrition, physical activity, and other lifestyle habits will help adults adopt healthy lifestyles early in their lives. As a result, $80 \%$ of resources spent in the last 6 months of life will occur when a person is 90 instead of 55-years-old.

Question, Tom Vogt, Kaiser Hawaii: In addition to the issues that you have raised, there are at least three other important issues that I would like your comments on. The first is that the perception of what's important to translate is often very different between clinicians and managers and researchers. The second is that from the organization standpoint, from the manager's or clinician's standpoint, when they perceive a problem, they want an answer quickly, and the timeframe often does not coincide with the grant application/funding timetable. The last problem is that when we set out to do research and evaluate something innovative that the system is doing, even if we have the money to collect the appropriate data, the system cannot resist the urge to modify as a study proceeds. The end result is that after completing a 2-year study, your evaluation is an evaluation of nothing real.

Mark Selna: At Geisinger, we wrestle with those issues all the time. We have years of longitudinal ambulatory data that have not been fully validated so there is a tremendous opportunity to conduct research with data that are already available. Also, if because of the topic or the clinician a particular study is best done very quickly, we try accommodating that need.

Question, Patrick O'Connor, HealthPartners: In the desire to provide 'perfect care', we are faced with new opportunities and new risks. It is a fact that all evidence-based findings do not have the same value or benefit to a given patient. Some evidence-based findings confirm much more benefit in terms of quality than others do, and yet all are evidence-based. With the new tools that we have, including electronic medical records and genetic information, the era of guidelines as we know them is going to be rapidly drawing to a close. The idea that a set of evidence-based guidelines for diabetes should be applied indiscriminately to all people with diabetes and called 'perfect care' is going to be replaced by personalized care. Ultimately, this approach would also conserve resources.

Brian Rank: One component of the research agenda is determining how we create that level of engagement with our patients about what is important.
Tom Kottke: "Personalized" health care will certainly be increasing as we discover genetic contributions to disease, but we cannot lose sight of the fact that there are certain things that everybody needs in their physical environment: clean air, clean water, high quality food, and freedom from physical harm. We also need to remember that the social environment has a powerful influence on disease experience. Unless we deal with physical and social environments, they will overwhelm everything we have to offer the patient as "personalized" care.

Douglas Reding: With all the information you have described, we need to rely on our electronic medical records with decision support to appropriately deal with the data. We need a real-time way to get information to help patients actually make the decisions about what kind of care they are willing to take, in the context of what sort of disease reduction rates they are willing to live with.

George Isham: We need to be thinking about getting upstream to the few problems that really make a difference, and about how to articulate that benefit.

Question, Justin Starren, Marshfield Clinic: If we actually succeed in figuring out how to improve care processes, how do we then share that knowledge in other settings?

George Isham: We need to think carefully about competitive advantage as a larger organization, as a not-for-profit organization with a research foundation in close proximity to delivery systems, for example. There are more ways to create a competitive advantage in a business sense than narrowly and assiduously protecting every shred of intellectual gain. Exploring that as larger organizations, and understanding the relationship between research organizations and our delivery system components, is a critical strategic issue for both research and delivery systems.

Question: Could you address the relationship between the patient and the doctor in terms of contributing to 'perfect care'?

Mark Selna: The social context is profound, but given the cost, we need to challenge ourselves with the incremental benefit of a doctor.

Douglas Reding: In terms of screening compliance for breast cancer, cervical cancer, or colon cancer, the data are clear that the doctor's directive for a patient to undergo screening is one of the highest predictors of whether or not a patient is compliant. The social context in which medicine is delivered is preeminent in terms of patient compliance. But given workforce shortages and cost issues, there have to be different ways and different models that can still effectively deliver the prevention message. 